\title{
Leukocyte Imaging of the Diabetic Foot
}

\author{
Chiara Lauri $^{1,2}$, Andor W.J.M. Glaudemans ${ }^{2}$ and Alberto Signore ${ }^{1,2, *}$
}

\begin{abstract}
${ }^{1}$ Nuclear Medicine Unit, Department of Medical-Surgical Sciences and of Translational Medicine, Faculty of Medicine and Psychology, "Sapienza" University of Rome, Italy; ' Department of Nuclear Medicine and Molecular Imaging, University of Groningen, University Medical Center Groningen, Groningen, The Netherlands
\end{abstract}

\begin{abstract}
Background: Diagnosing diabetic foot infection is often difficult, despite several available diagnostic methods. Amongst these, several imaging modalities exist to evaluate the diabetic foot in case of a suspected osteomyelitis. Nuclear Medicine, in particular, offers a variety of radiopharmaceuticals and techniques. Nowadays the gold standard radionuclide procedure, when an osteomyelitis is suspected, is represented by the use of radiolabelled leukocytes with either ${ }^{99 \mathrm{~m}} \mathrm{Tc}-\mathrm{HMPAO}$ or ${ }^{111} \mathrm{In}$-oxine.

Methods: In this review, we describe the correct acquisition and interpretation of white blood cell scintigraphy and we provide an overview of the existing literature data of the use of this technique in the infected diabetic foot. If images are correctly acquired, displayed and interpreted, this modality reaches very high diagnostic accuracy $(>95 \%)$ in detecting osteomyelitis and it allows the differential diagnosis with a soft tissue infection or inflammation. Single-photon emission computed tomography/computed tomography (SPECT/CT) in addition to planar images is mandatory to determine the extent and exact location of the infective process in both fore foot and midhint foot. With the addition of bone marrow scintigraphy using radiolabelled nanocolloids, radiolabelled white blood cell scintigraphy is also able to differentiate between Charcot neuroarthropathy and osteomyelitis, which is a challenge in the evaluation of diabetic foot. Radiolabelled anti-granulocyte monoclonal antibodies and their fragments can also be used instead of white blood cells although there is a limited experience on their usefulness in diabetic foot infection.
\end{abstract}

A R T I C L E H IS T O RY

Received: February 1, 2018 DOI:

Keywords: Diabetic foot, WBC scintigraphy, radiolabelled leukocytes, nuclear medicine, infection, osteomyelitis, diagnosis.

\section{INTRODUCTION}

It has been estimated that approximately $15-20 \%$ of all persons suffering from diabetes will develop a pedal ulcer during the course of the disease and about a quarter of these patients will require amputation [1-3]. Thus, diabetes represents the principal indication to non-traumatic amputation. The ulcers may remain unrecognized for a long period due to the concomitant peripheral neuropathy, that is usually present in non-controlled diabetes, and this condition together with vascular insufficiency, repeated biomechanical stress and local trauma, can favour the colonization of wounds by several micro-organisms. The ulcer, indeed, is the primum movens for the development of an infection that primary invades the soft tissue which can be followed by spreading to the underlying bone, causing osteomyelitis (OM) [4, 5]. Diabetic foot osteomyelitis (DFO) must be distinguished from Charcot foot, a condition of neuroosteoarthropathy that affects approximately $2.5 \%$ of the diabetic population. It is characterized by a progressive degeneration of the musculoskeletal system of primarily the tarsal-metatarsal joints causing bone deformities and disruption of foot architecture [6]. While Charcot foot only requires immobilization, in the presence of infection the treatment strongly depends on the extent of the infection and in particular if it involves only soft tissue or also the bone. Therapeutic options can vary from a watchful waiting approach to re-vascularization or antibiotic treatment or debridement depending on the cases. The prevention of ulcers and a prompt diagnosis of infection are critical aspects in order to ensure an appropriate and a non-invasive treatment aiming to avoid the amputation.

*Address correspondence to this author at the Nuclear Medicine Unit, Department of Medical-Surgical Sciences and of Translational Medicine, "Sapienza" University of Rome, Ospedale S. Andrea, Via di Grottarossa 1035, 00189 Rome, Italy; E-mail: alberto.signore@uniroma1.it
Early diagnosis of DFO is often problematic, as it is difficult to differentiate between OM, soft tissue infection, inflammation or Charcot. There are two major problems that are the cause for this difficult diagnosis. Firstly, all open wounds are colonized with micro-organisms, making culture results often non-definitive. Secondly, the presence of the earlier mentioned peripheral neuropathy and vascular disease can either diminish or mimic inflammatory findings, both in the soft tissue and underlying bones, reducing their usefulness.

Local signs and symptoms and biochemical markers of flogosis are not specific for OM, so the appeal to microbiology and histopathology is mandatory. Nevertheless, bone biopsy is an invasive procedure; it needs to be aimed at the target area and may result in sample errors due to contamination of the bone biopsy by superficial soft tissue infection. The probe-to-bone test can be helpful in diagnosing DFO, but only if it is performed and interpreted well. Sensitivity and specificity of the probe-to-bone test may be high in experienced hands, but results are varying widely. Laboratory values such as the erythrocyte sedimentation rate (ESR) and C-reactive protein (CRP) have been addressed; their lack of specificity has proved to constitute a substantial issue. Existing imaging modalities offer a wide panel of choices as alternative or in conjunction to it. The role of imaging in diagnosing and managing DFO is expanding and often crucial. The aims of all existing imaging techniques include helping to either exclude an infection or to confirm the diagnosis, to evaluate the extent of an existing infection, and to differentiate between $\mathrm{OM}$, soft tissue infection and neuro-osteoarthropathy. Nowadays, there is a huge variety in used diagnostic imaging techniques in patients with DFO amongst different centres. Common agreement is to start with plain X-rays. Despite radiology offers high quality images of CT and MRI, Nuclear Medicine (NM) with its functional information, can be considered the first diagnos- 
tic approach in most cases. Furthermore, NM modalities, and radiolabelled white blood cell scintigraphy (WBCs) in particular, are able to promptly recognize a bone infection and are able to discriminate this condition from a sterile inflammation or a soft tissue infection. This modality could be very useful also in the follow-up of antibiotic therapy, documenting the resolution or the persistence of disease. The diagnostic accuracy of WBCs is not affected by patient's glycaemia or antibiotic treatment.

In the next sections we will explore the feasibility of leukocyte imaging in DFO with a particular emphasis to WBCs, which is considered the gold standard NM examination for the detection of an infection.

\section{IN VITRO LABELLED LEUKOCYTE IMAGING}

Radiolabelled WBCs have been introduced since 1970s for the diagnosis of infection and it is nowadays a consolidated methodology that can be applied in this field, being the NM gold standard when an infective process is suspected. This modality specifically targets activated granulocytes, mainly neutrophils, whose migration over time in peripheral sites represents a surrogate marker of bacterial infection [7].

\subsection{Preparation}

The labelling procedure must be performed in accordance to the recommendations of European Association of Nuclear Medicine (EANM) $[8,9]$. For this purpose $30-40 \mathrm{ml}$ of blood of the patient must be collected. After the in vitro separation of leukocytes by red cell sedimentation, granulocytes are radiolabelled and then reinjected into the patient. Both ${ }^{99 \mathrm{~m}} \mathrm{Tc}$ - hexamethylpropylene amine oxime (HMPAO) and ${ }^{111}$ In-oxine can be used for the labelling procedure. The first agent is generally preferable considering the favourable physical characteristics that allow high quality images with the gamma-camera, the shorter half-life and the lower radiation burden compared to ${ }^{111}$ In. Despite these advantages, the use of ${ }^{99 \mathrm{~m}}$ Tc-HMPAO has a major limitation studying abdominal pathologies due to its early excretion by the bowel. ${ }^{111}$ In-oxine can be preferable in these circumstances. Moreover the use of ${ }^{111}$ In-oxine is preferable in chronic infections or when very late images (after 24 hours) are required [10].

Independently by the isotope used, the labelling of leukocytes is a time consuming procedure (usually 60-90 $\mathrm{min}$ ) that requires an adequate laboratory equipped with centrifuges and a laminar flow cabinet (class A) or an isolator, in order to ensure sterility during the labelling, despite the availability of disposable sterile kits [11]. Trained personnel is necessary to perform the labelling procedure as it has some risk related to the manipulation of radioactive blood that could also be potentially infected. Finally, each centre has to validate the procedure and quality controls and these validation tests must be performed annually.

\subsection{Imaging Protocol and Interpretation Criteria}

Data in literature regarding the diagnostic accuracy of radiolabelled WBCs scan in acute OM or prosthetic joint infections are extremely variegated and they are strongly dependant on the protocol of image acquisition. In some cases they reach more than $90 \%$ of accuracy [12-14, 17]. This discrepancy mainly depends on the different acquisition protocols and interpretative criteria adopted [15-17]. The correct imaging protocol should be based on the knowledge of pathophysiology of infection and it should take into account that in acute, sub-acute and chronic infections the recruitment of granulocytes, from blood to the affected site, is a continuous dynamic process over time mainly induced by the production of chemotactic factors from bacteria. In sterile inflammatory conditions, the migration of granulocytes, from blood to lesion, is usually transient. For this reason a single set of images at a fixed time point is not sufficient to differentiate an infection from a sterile inflammation, and at least two images acquired at two different time points (typically 3 hours and 20 hours post injection) are necessary to detect soft tissue infections (STI) or bone infections. Early images (30 minutes-1 hour post injection) can be a useful map of bone marrow activity.

In case of diabetic osteomyelitis, the situation is even more complicated due to the presence of micro- and macro-angiopathy. Therefore, the granulocyte migration from blood to lesion is usually impaired and granulocytes do not migrate sufficiently over time to compensate isotope decay over time. As a result, a soft tissue infection in the fore foot is usually not seen with an increase of activity over time and cannot be distinguished from a sterile inflammatory lesion. Bone infections, being usually more vascularised, still show an increased accumulation of granulocytes over time, and can therefore be diagnosed with standard interpretation criteria (see Fig. 1).

Each set of images should be acquired with an acquisition time corrected for the decay of the isotope. In a large multicentre study published in 2013, the authors compared different acquisition pro-

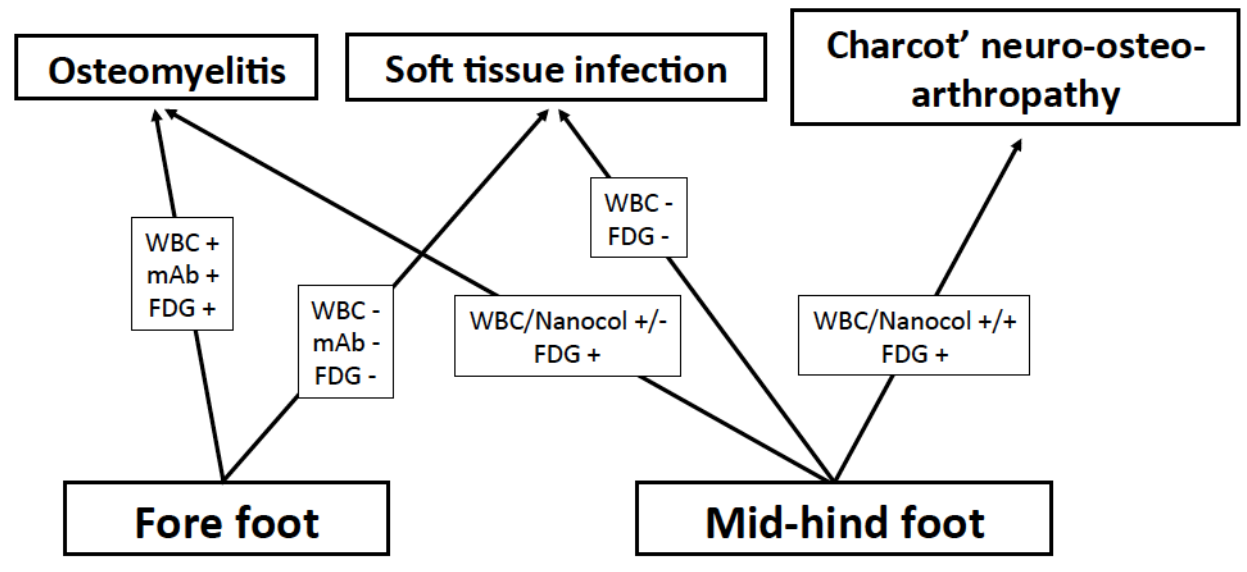

Fig. (1). Diagnostic flow chart for the correct use of nuclear medicine techniques and radiopharmaceuticals in the diagnosis in diabetic foot infection. In case of suspected fore foot infection both $\mathrm{WBC}, \mathrm{mAb}$ and FDG can differentiate between osteomyelitis and soft tissue infection mainly because of the use of hybrid imaging. In case of suspected mid-hind foot infection FDG cannot differentiate between an osteomyelitis and a Charcot neuroarthropathy, while WBC in conjunction with bone marrow scintigraphy with nanocolloids can allow the differential diagnosis between osteomyelitis, soft tissue infection or Charcot neuroarthropathy. 
tocols and interpretative criteria and they finally concluded that the highest diagnostic performance of WBCs scan is obtained when a three time point acquisition protocol is used $\left(30^{\prime}-1 \mathrm{hr}, 3-4 \mathrm{hrs}, 20\right.$ $24 \mathrm{hrs}$ post injection) with an acquisition time corrected for the isotope decay $[15,17]$. Once acquired, the images should be correctly displayed in absolute counts or equalizing bone marrow as a reference tissue and using the same intensity colour scale in order to avoid any operator bias.

The interpretation can be performed visually (qualitative analysis) or using a semi-quantitative analysis. Using the first method, a scan is considered positive for infection if an increased uptake over time, in terms of intensity and/or extension, is observed in the affected site, in respect to the contralateral comparing delayed and late images whereas, in presence of a decreased uptake over time, the diagnosis of infection can be ruled out. In equivocal cases a semi-quantitative analysis can be very helpful drawing region of interests (ROIs) on the affected site and contralateral reference tissue and comparing the target-to-background ratio (T/B) of delayed and late images. Also here, when the T/B ratio is increasing in time, the scan is considered positive, when the T/B ratio is decreasing in time, the scan is concluded to be negative for an infection.

\subsection{WBCs Scintigraphy in the Evaluation of Diabetic Foot, Analysis of Literature}

In a recently published meta-analysis and systematic review based on studies comparing the diagnostic performance of different imaging modalities for diagnosis of DFO (WBCs, ${ }^{18}[\mathrm{~F}]-\mathrm{FDG}-$ $\mathrm{PET} / \mathrm{CT}$ and MRI) it emerges that the pooled sensitivity and specificity of WBC scintigraphy were respectively $92 \%$ and $75 \%$ for ${ }^{111}$ In-oxine and $91 \%$ and $92 \%$ for ${ }^{99 m}$ Tc-HMPAO [18]. Comparing the performance of these four imaging modalities, ${ }^{99 \mathrm{~m}} \mathrm{Tc}-\mathrm{HMPAO}$ WBCs has the highest specificity followed by ${ }^{18}[$ F]-FDG-PET/CT, MRI and ${ }^{111}$ In-oxine leukocytes scintigraphy whereas the sensitivity was approximately $90 \%$ for all imaging modalities [18]. Furthermore, the majority of the studies with ${ }^{99 \mathrm{~m}} \mathrm{Tc}$-HMPAO WBCs were performed only with planar imaging. As we will stated later in this article, SPECT/CT has many advantages and leads to higher diagnostic accuracy. Therefore, it can be expected that the specificity for ${ }^{99 \mathrm{~m}} \mathrm{Tc}-\mathrm{HMPAO}$ WBCs with SPECT/CT even leads to higher results. Furthermore, as will be explained more extensively later on, not all studies used the above mentioned correct acquisition protocols. Therefore, despite the already high specificity, further improvement of the used techniques in WBCs definitely leads to even better results.

The systematic analysis of literature regarding the application of WBCs scintigraphy in the detection of DFO shows very discordant results. The reported sensitivity and specificity range from $75 \%$ [19] to $100 \%$ [20-22] and from $67 \%$ [22] to $100 \%$ [23], respectively. The reason of these huge variation in results can be explained by the different acquisition protocols and interpretation criteria adopted among the studies that makes it very difficult to perform a direct head to head comparison of the studies. Some authors used only one step images after 2 hours [24], or 3 hours [25-28], or 4 hours [29, 30], or even 24 hours post injection [22, 3134]. Some others acquired at two-time point protocol with images after 4 and 24 hours post injection [35, 36]. Furthermore, these images were acquired by fixed time (e.g. 10 minutes), fixed number of counts or with decay-corrected acquisition times but only this last protocol is correct. In many cases the acquisition protocols are not even mentioned in methodology further complicating the comparison of sensitivity, specificity and diagnostic accuracy between the studies. Moreover, it is reasonable to hypothesize that the lowest performance of WBCs scintigraphy that we can find in some studies $[25,30,33]$ can be correlated to out dated protocols of acquisition.
Only two groups acquired early, delayed and late images [37, 38]. Filippi et al. in 2009 acquired planar images after 30', 4 hours and 24 hours but used fixed acquisition times per each image (10' at $30^{\prime}$ and 4 hours, and $15^{\prime}$ at 24 hours) and adopting a qualitative interpretation analysis, comparing the affected foot with the contralateral. They also performed a SPECT/CT after 6 hour improving the diagnostic accuracy of WBCs scan [37]. Familiari et al. also acquired three sets of images (early, delayed and late) with acquisition times corrected for ${ }^{99 \mathrm{~m}} \mathrm{Tc}$ decay and reproducing the images in monitors in absolute counts with the same intensity colour scale [38]. The qualitative analysis gave a diagnostic accuracy of $92 \%$. Using semi-quantitative analysis the best results were obtained when the following criteria were adopted: the scan was considered positive for infection when T/B ratio after 20 hours was greater than 2.0 and the ratios increased over time; the scan was classified as negative for $\mathrm{OM}$ when $\mathrm{T} / \mathrm{B}$ ratio after 20 hours was less than 2.0 and the ratios decreased over time or remained stable; the scintigraphy was considerate indicative for soft tissue infection when the $\mathrm{T} / \mathrm{B}$ ratio after 20 was greater than 2.0 and the ratios decreased over time or remained stable. Using these interpretative criteria, the sensitivity, specificity, positive predictive value, negative predictive value and diagnostic accuracy of ${ }^{99 \mathrm{~m}} \mathrm{Tc}-\mathrm{HMPAO}$ WBCs scintigraphy were $86 \%, 100 \%, 100 \%, 86 \%$ and $92 \%$, respectively. These results stress the concept that DFO is a particular form of OM that cannot be approached as a common OM. In DF the reduced blood supply to peripheral tissue due to vascular insufficiency, is maybe responsible of the typical behaviour of soft tissue infection that show a decreased uptake over time (like aseptic flogosis) whereas only OM shows an increased uptake over time, as mentioned before. This phenomenon can be evident only if the images are well acquired (using time corrected for isotope decay), displayed (using absolute counts and not the percentage of maximum pixel), and interpreted (considering the behaviour and the uptake over time), (see Fig. 1).

Another important aspect that need to be stressed and that further complicates the scenario of DF, is that disorders of forefoot are different, and require a different approach, from the disorders of mid-and hindfoot [3]. In forefoot disorders WBCs scintigraphy is able to achieve a precise differential diagnosis between $\mathrm{OM}$ and soft tissue infection using the precautions previously described. In the analysis of mid- and hindfoot the eventual presence of Charcot neuro-osteo-artropathy must be also taken in consideration, thereby further complicating the differential diagnosis. This condition is associated with the disruption of bony architecture and WBC uptake in this specific situation may reflect the bone marrow expansion due to inflammatory response [39-41] resulting in a reduced specificity of WBCs in this region compared with forefoot. In order to differentiate a real infection from a non-specific bone marrow expansion and to improve the specificity of radiolabelled leukocytes scintigraphy, it is suggested to perform a combined WBCs scan and bone marrow scintigraphy using radiocolloids. Both radiopharmaceuticals accumulate in bone marrow but, in presence of infection, only WBCs scan is positive. Therefore, if the images of both examinations are not congruent (positive at WBC, negative at colloids) we are in presence of OM, on the contrary if the results of these two examinations are concordant (presence of uptake at both WBCs scan and nanocolloids), the infection can be ruled out and the diagnosis of Charcot foot can be formulated [3, 39-41]. These concepts are resumed in Fig. 1 and Fig. 2.

\subsection{Added Value of Hybrid Images (SPECT-SPECT/CT)}

The main disadvantage of SPECT nuclear medicine imaging techniques is represented by the limited spatial resolution and the lack of anatomic localisation, which is especially a problem in the foot with on 2D planar imaging overlapping of all the bony structures. Therefore, the appeal to hybrid images (i.e. combination of physiological nuclear medicine imaging and anatomic radiological 

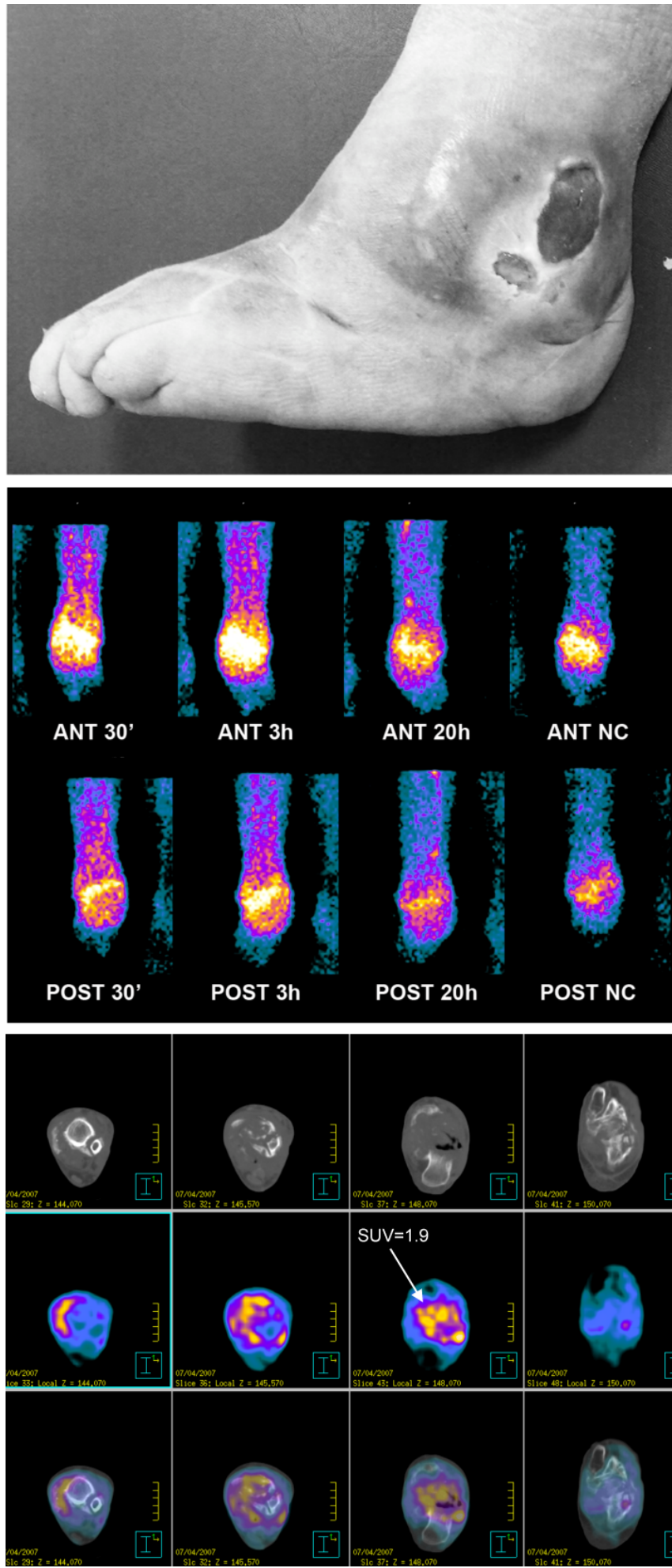

Fig. (2). A case of suspected osteomyelitis in a diabetic patient with a skin ulcer and a Charcot osteo-arthropathy of mid-hind foot (top image). A ${ }^{99 \mathrm{~m}}$ Tc-HMPAO-WBC scan was performed in this patient with images at 30'$3 \mathrm{~h}-20 \mathrm{~h}$ showing an accumulation of labelled cells in the ankle, although decreasing in intensity between $3 \mathrm{~h}$ and $20 \mathrm{~h}$ (middle panel of images). The result of WBC scan suggested a sterile inflammation (due to Charcot osteoarthropathy) without osteomyelitis. The nanocolloid (NC) image was matching with the $20 \mathrm{~h}$ WBC image thus confirming the presence of a Charcot lesion without osteomyelitis. The bottom panel of images show the $\left[{ }^{18} \mathrm{~F}\right] \mathrm{FDG}$ PET/CT transaxial sections of the same patient also confirming an inflammatory/degenerative disease $\left(\mathrm{SUV}_{\max }=1.9\right)$. imaging), when available, is mandatory in order to improve the diagnostic accuracy of planar images. The incremental value of SPECT and SPECT/CT is indisputable. The fusion of functional information of NM examinations with anatomical landmarks provided by $\mathrm{CT}$ scan has changed the way of make imaging in several fields by obtaining high quality images, especially when a contrast agent is administered during the CT scan.

Several authors explored the possible application of SPECT/CT in the diagnosis $[24,25,31,32,37]$ and therapy monitoring of DFO $[42,43]$. Although they used different protocols of acquisition they all concluded that this hybrid imaging modality improves the diagnosis through a better localization of the infective foci into bone or soft tissue with an excellent definition of the extent of the process. In the series of patients retrospectively studied by Przybylski et al. the sensitivity, specificity and diagnostic accuracy of ${ }^{99 \mathrm{~m}} \mathrm{Tc}$ WBC scintigraphy were $87.5 \%, 71.4 \%$ and 80 respectively [25]. Similar findings occurred in the large population of 272 patients examined by Heiba et al. using a protocol combining ${ }^{111}$ In WBC scan and bone scan [31]. They compared the diagnostic value of dual isotope SPECT/CT ( ${ }^{11}$ In WBC + bone scintigraphy) with the results obtained from SPECT/CT of WBCs and bone scan alone. Considering the small size of bony structures in the foot, an uptake on the soft tissue at planar images may overlap the underlying bone and vice versa leading to a wrong interpretation of the exam and a consequent erroneous treatment. In their series dual isotope SPECT/CT was superior to bone scan or WBCs with SPECT/CT alone in discriminating $\mathrm{OM}$ from soft tissue infection and in defining the exact extent of the process. The same authors also found that the use of dual isotope SPECT/CT is associated with a reduced length of hospitalization compared with conventional modalities and it also could be beneficial in the optic of limb salvage procedures [32]. Erdman et al. proposed a severity index based on the combination of intensity of uptake (compared to blood vessels), stage (involvement of bone cortex or marrow) and number of lesions [24]. They concluded that this score system is more accurate than the conventional interpretative method and that it could be able to predict the outcome and to select patients candidates to a prolonged antibiotic therapy. However, the conventional interpretative criteria adopted were only based on a qualitative evaluation of the uptake on the bony structures and it did not consider the increase of activity over time thus introducing a bias. Filippi et al. in 2009 used three-time point acquisition combined with performing a SPECT/CT 6 hours post injection [37]. In $52.6 \%$ of cases the interpretation of the examination was substantially changed on the basis of SPECT/CT, being able to rule out the infection in 6 cases, to diagnose OM in 1 case and to better define the extent of the process in 3 cases.

We can conclude that, despite the heterogeneity in used acquisition protocols and interpretation criteria, data in literature support the use of SPECT/CT in addition to planar images in the evaluation of DFO, especially in positive scans in order to better define the localization of the infection (in or outside the bone) and the extent of infection. Two clinical examples of the added value of hybrid imaging in DFO are shown in Figs. 3 and 4.

\subsection{Role of WBC in Therapy Monitoring}

Another additional potential use of WBCs with SPECT/CT is in helping to determine when DFO has resolved. This is a major issue in patients with DFO, since there is no general rule for how long antibiotic treatment should be prolonged, when it can be stopped, or whether or not surgical treatment is needed when antibiotic treatment is not helping.

Some authors explored the application of WBC scintigraphy in the evaluation of antibiotic treatment response [35, 36, 42, 43] but data in literature are mainly based on small series and do not allow to draw a definitive conclusion. However, preliminary findings seem to support the utility of this modality to document the resolu- 

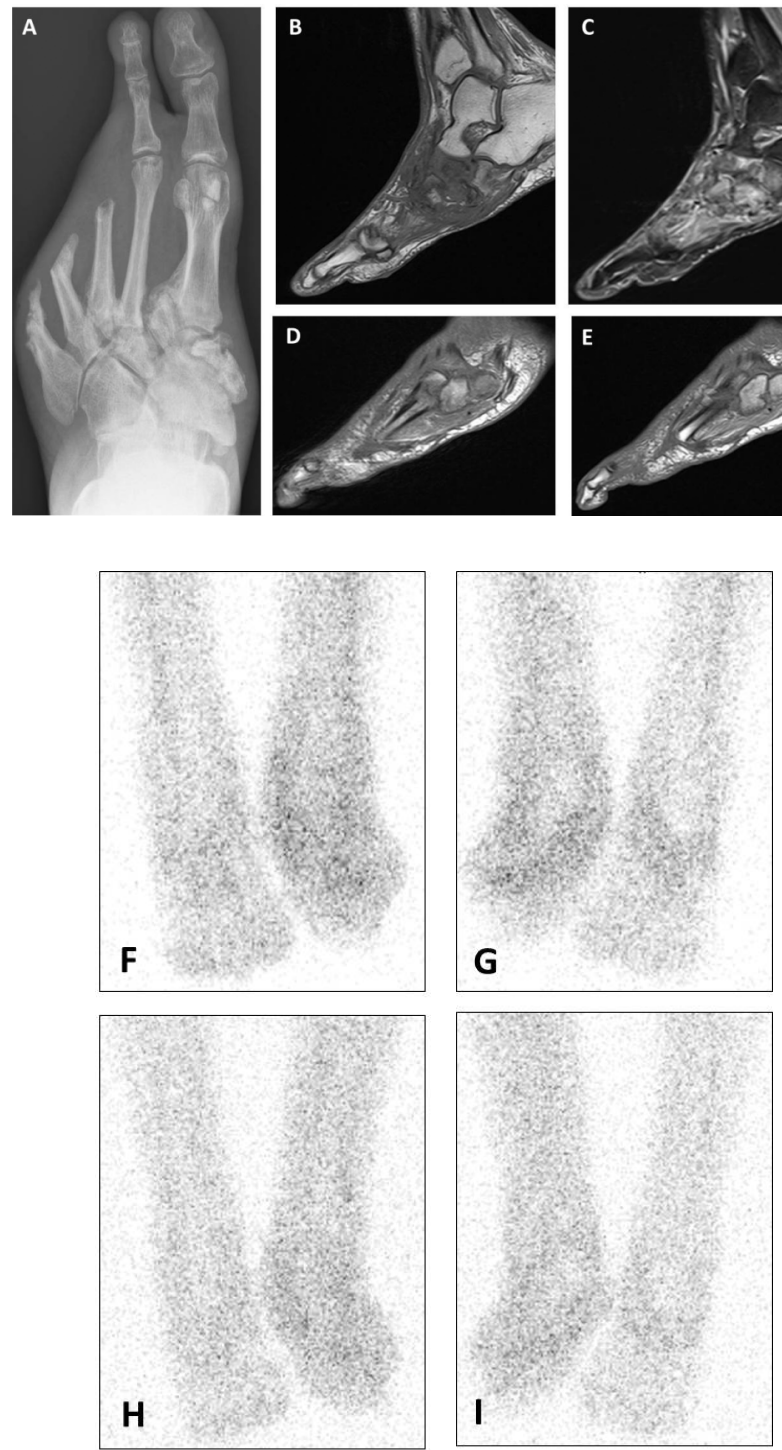

Fig. (3). A case of a 52-year-old male patient with diabetes. One year ago amputation tarsometarsal joints of the $3^{\text {rd }}, 4^{\text {th }}$ and $5^{\text {th }}$ toe. He now presented with a new ulcus at the base of metatarsal 5 . The question from the referring clinician was if there was an osteomyelitis or a Charcot foot. (A) X-ray showing destruction of the cuneiform bones and an irregular cortex at the amputation side of metatarsal 5; could be Charcot and a possible underlying osteomyelitis. (B) MRI, T1-weighted image showing a decreased signal intensity in the midfoot; (C-D-E) MRI, TIRM (turbo intensity recovery magnitude) sequence showing an irregular aspect of metatarsal 5 and a hyper intense signal at the distal part of metatarsal 5 and fat induration. Again no clear diagnosis; could be Charcot with possible osteomyelitis. (FG) White blood cell scintigraphy, anterior and posterior images after $4 \mathrm{~h}$; (H-I) White blood cell scintigraphy, anterior and posterior images after $20 \mathrm{~h}$ : no increase in uptake with time, so no osteomyelitis. Final diagnosis: Charcot foot.

tion of infection after an antibiotic treatment with a sensitivity and specificity superior than MRI in detecting residual disease [35]. Newman et al. in 1991 performed ${ }^{111}$ In WBC scan at 2-to 3-week intervals during antibiotic treatment and they found that the intensity of the uptake decreases after 16-34 days of antimicrobial treatment and normalizes after 36-54 days [36]. Vouillarmet et al. performed ${ }^{99 \mathrm{~m}} \mathrm{Tc}$ WBC SPECT/CT to assess the evolution of OM after the treatment and they also obtained very high sensitivity, speci-
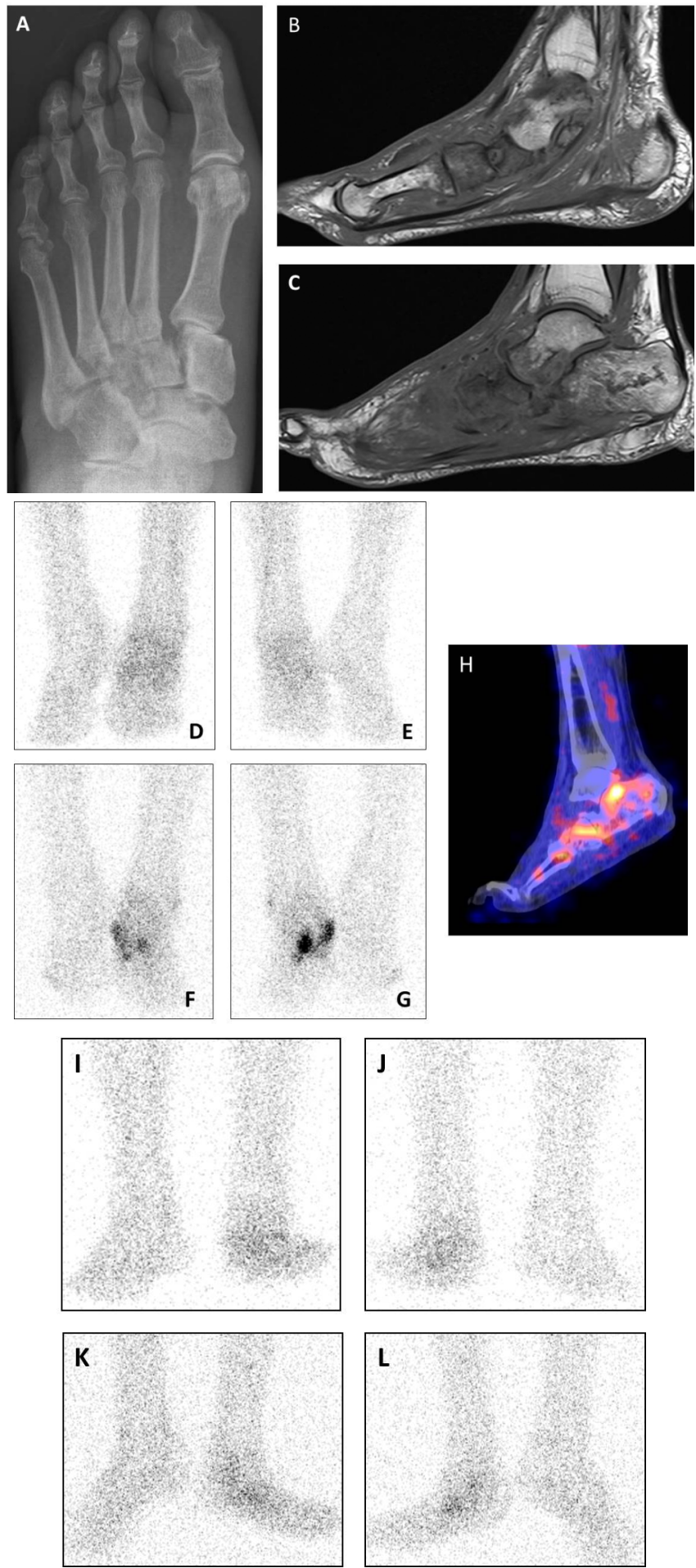

Fig. (4). A case of a 65-year-old male patient with diabetes since the age of 20. Known with polyneuropathy and a Charcot foot since 3 years. Now abscess left foot plantar side. (A) X-ray showing irregular cortices of the tarsalia, no other problems. (B-C) MRI, T1-weighted sequences showing a low signal intensity in the navicular bone, cuneiform bones, anterior part of the calcaneus, and the cuboid bone. Could be osteomyelitis or Charcot. (DE) White blood cell scintigraphy, anterior and posterior images after $4 \mathrm{~h}$; (FG) White blood cell scintigraphy, anterior and posterior images after $20 \mathrm{~h}$ : increase in uptake in time, at SPECT-CT (performed after $4 \mathrm{~h}$, image $\mathrm{H}$ ) located at the anterior part of the calcaneus and in the cuboid bone. Final diagnosis: osteomyelitis. (I-L) white blood cell scintigraphy performed after 6 weeks of antibiotic treatment, anterior and posterior images after 4 and 20 $\mathrm{h}$ : all negative, so no infection anymore. 
ficity, PPV and NPV being respectively of $100 \%, 91.5 \%, 71.5 \%$ and $100 \%$ [42]. In a recent retrospective study performed by Lazaga et al. the sensitivity, specificity, PPV, NPV of ${ }^{99 m} \mathrm{Tc}$ WBC SPECT/CT in determining OM remission were $90 \%, 56 \%, 69 \%$ and $83 \%$ respectively being able to determine the outcome of DFO [43].

The issue that on-going antibiotic treatment could influence the sensitivity of WBC scan is still debated and the opinions are very contrasting so it is not possible to define the perfect timing to perform WBCs scan following antimicrobial therapy. However, from preliminary findings it seems that the diagnostic power of $\mathrm{WBC}$ is not significantly affected by the administration of antibiotic $[35,36$, $42,43]$. Although not focused on DFO, in a large population of patients with OM, retrospectively studied by Glaudemans et al., it emerges that there were no significant differences in terms of diagnostic performance between patients under treatment and patients that were not receiving therapy, thus supporting the idea that if correctly acquired, displayed and interpreted, WBCs retains a high sensitivity and specificity in detecting residual disease independently by the administration of antibiotics [15].

\section{IN VIVO LABELLED LEUKOCYTES IMAGING}

In order to overcome the time-consuming and possible hazards of the labelling procedure of WBCs, several efforts have been directed towards the development of alternative easier solutions but with preservation of the same diagnostic accuracy. The most successful approach is the production of monoclonal antibodies $(\mathrm{MoAb})$ directed against specific antigens expressed on the surface of activated granulocytes. Despite useful in most cases of infection, they have some limitations. First of all the high molecular weigh of the entire antibodies represents a (2) stacle to their diffusion into the site of infection (which can me a thajor problem in the diabetic foot with often vascular impairment), secondly they have a long plasma half-life, and thirdly they have a clearance by the reticuloendothelial system so they show high uptake in liver and bone marrow. In order to have a good T/B ratio, the study should be performed after 6 hours post injection and should be repeated after 24 hours [44]. Moreover, the majority of the molecules accumulate into inflamed site in a non-specific way, as a result of increased vascular permeability. Another important limitation to the use of $\mathrm{MoAb}$ is the possibility to induce human murine antibodies (HAMA) in the host that represents an important obstacle in their possible application in follow-up. Indeed, injecting a MoAb in a patient with HAMA leads to an alteration of the biodistribution of the molecule thereby degrading the quality of the images and the diagnostic accuracy.

In order to overcome the limitations related to the entire antibody, the use of antibody fragments (Fab') has been proposed as an alternative. Due to the low weight, they present a higher accumulation in infected tissue, a faster clearance and a lower immunogenicity compared to MoAb [15] but, unfortunately, also the diagnostic accuracy is somewhat lower.

\subsection{Monoclonal Antibodies and their Fragments in the Evalua- tion of Diabetic Foot}

Several authors have investigated the use of MoAb or Fab' fragments in DF. In 1993 some authors explored the utility of Besilesomab (Scintimun ${ }^{\circledR}$ ), a ${ }^{99 \mathrm{~m}}$ Tc-labelled anti-granulocyte antibody directed against an antigen called NCA-95 expressed on neutrophils, in the evaluation of 25 patients with a suspected DFO, reporting a sensitivity, specificity and diagnostic accuracy of $93 \%, 78 \%$ and $84 \%$ [45]. Palestro and co-workers used ${ }^{99 \mathrm{~m}} \mathrm{Tc}$-Fanolesomab (LeuTech $\AA$ ), a monoclonal murine immunoglobulin that binds to CD15 on neutrophils, in 25 patients affected by pedal ulcers [46]. The same patients were also studied with ${ }^{111}$ In-WBCs and three phase bone scan. The authors found similar results between the in vivo labelled leukocytes $\left({ }^{99 \mathrm{~m}} \mathrm{Tc}\right.$-Fanolesomab) and the in vitro labelled WBCs $\left({ }^{111} \mathrm{In}-\mathrm{WBCs}\right)$ in terms of sensitivity, specificity and diagnostic accuracy being respectively $90 \%$ vs $80 \%, 67 \%$ vs $67 \%$ and $76 \%$ vs $72 \%$.

Some authors tried to assess the utility of antibody fragments in DF evaluation, using in particular Sulesomab (Leukoscan ${ }^{\circledR}$ ), a murine monoclonal antibody fragment of the IgG1 class directed against NCA-90 of neutrophils. Delcourt and colleagues compared combined Fab'/bone scan with combined gallium/bone scan in 31 sites of suspected OM reporting higher diagnostic performance of the first imaging modality [47]. Harwood et al. experimented the feasibility of Sulesomab in a large population of 122 diabetic patients with foot ulcers confirming the high diagnostic performance of this modality that could be used in alternative to in vitro labelled leukocytes.

Not many others have confirmed these results and therefore the use of MoAb or Fab' in DF remains a second choice, being WBCs with SPECT/CT and FDG-PET/CT first options in terms of diagnostic accuracy $[18,49,50]$. Other new radiopharmaceuticals are now available for imaging bacteria with hopes they can provide useful additional information when combined with WBC scan [51].

\section{CONCLUSION}

Evaluation of an infection in patients with a diabetic foot is sometimes still a challenge for clinicians. A prompt identification of infection is however mandatory in order to ensure a fast and best treatment for the patients. The choice of the most appropriate imaging modality needs an evaluation of clinical presentation, real indication to the exam and the results of previously performed tests. Radiolabelled WBC scan should always be considered in the first diagnostic steps as it retains a very high diagnostic performance as it specifically shows the migration of activated granulocytes in infected sites, representing a surrogate marker of an infection. The diagnostic accuracy of this modality strongly depends on the image acquisition protocol and on the interpretation criteria adopted. Therefore, to present uniform results and to have highest diagnostic accuracy, further studies should really be performed conform the EANM guidelines for WBCs. SPECT/CT is also recommended, when available, in order to better define the exact location (in or outside the bone) and the extent of infection.

\section{CONSENT FOR PUBLICATION}

Not applicable.

\section{CONFLICT OF INTEREST}

The authors declare no conflict of interest, financial or otherwise.

\section{ACKNOWLEDGEMENTS}

Declared none.

\section{REFERENCES}

[1] Ramsey SD, Newton K, Blough D, et al. Incidence, outcomes, and cost of foot ulcers in patients with diabetes. Diabetes Care 1999; 22(3): 382-7.

[2] Reiber GE, Lipsky BA, Gibbons GW. The burden of diabetic foot ulcers. Am J Surg 1998; 176(2A)(Suppl.): 5S-10S.

[3] Capriotti G, Chianelli M, Signore A. Nuclear medicine imaging of diabetic foot infection: results of meta-analysis. Nucl Med Commun 2006; 27(10): 757-64.

[4] Prompers L, Huijberts M, Apelqvist J, et al. High prevalence of ischaemia, infection and serious comorbidity in patients with diabetic foot disease in Europe. Baseline results from the Eurodiale study. Diabetologia 2007; 50(1): 18-25.

[5] Lipsky BA. Osteomyelitis of the foot in diabetic patients. Clin Infect Dis 1997; 25(6): 1318-26.

[6] Gierbolini R. Charcot's foot: often overlooked complication of diabetes. JAAPA 1999; 12(6): 62-8.

[7] Signore A, Lauri C, Galli F. Radiolabelled probes targeting infection and inflammation for personalized medicine. Curr Pharm Des 2014; 20(14): 2338-45. 
[8] Roca M, de Vries EF, Jamar F, Israel O, Signore A. Guidelines for the labelling of leucocytes with (111)In-oxine. Eur J Nucl Med Mol Imaging 2010; 37(4): 835-41.

[9] de Vries EF, Roca M, Jamar F, Israel O, Signore A. Guidelines for the labelling of leucocytes with $(99 \mathrm{~m}) \mathrm{Tc}-\mathrm{HMPAO}$. Eur J Nucl Med Mol Imaging 2010; 37(4): 842-8.

[10] Peters AM. The utility of [99mTc]HMPAO-leukocytes for imaging infection. Semin Nucl Med 1994; 24(2): 110-27.

[11] Signore A, Glaudemans AW, Malviya G, et al. Development and testing of a new disposable sterile device for labelling white blood cells. Q J Nucl Med Mol Imaging 2012; 56(4): 400-8.

[12] Palestro CJ, Love C, Bhargava KK. Labeled leukocyte imaging: current status and future directions. Q J Nucl Med Mol Imaging 2009; 53(1): 105-23.

[13] Love C, Marwin SE, Palestro CJ. Nuclear medicine and the infected joint replacement. Semin Nucl Med 2009; 39(1): 66-78.

[14] Gemmel F, Van den Wyngaert H, Love C, Welling MM, Gemmel $\mathrm{P}$, Palestro CJ. Prosthetic joint infections: radionuclide state-of-theart imaging. Eur J Nucl Med Mol Imaging 2012; 39(5): 892-909.

[15] Glaudemans AW, Galli F, Pacilio M, Signore A. Leukocyte and bacteria imaging in prosthetic joint infection. Eur Cell Mater 2013; 25: 61-77.

[16] Tondeur MC, Sand A, Ham HH. Interobserver reproducibility in the interpretation of $99 \mathrm{mTc}$-labelled white blood cell scintigraphic images. Nucl Med Commun 2008; 29(12): 1093-9.

[17] Erba PA, Glaudemans AW, Veltman NC, et al. Image acquisition and interpretation criteria for 99mTc-HMPAO-labelled white blood cell scintigraphy: results of a multicentre study. Eur J Nucl Med Mol Imaging 2014; 41(4): 615-23.

[18] Lauri C, Tamminga M, Glaudemans AWJM, et al. Detection of Osteomyelitis in the Diabetic Foot by Imaging Techniques: A Systematic Review and Meta-analysis Comparing MRI, White Blood Cell Scintigraphy, and FDG-PET. Diabetes Care 2017; 40(8): 1111-20.

[19] Maurer AH, Millmond SH, Knight LC, et al. Infection in diabetic osteoarthropathy: use of indium-labeled leukocytes for diagnosis. Radiology 1986; 161(1): 221-5.

[20] Keenan AM, Tindel NL, Alavi A. Diagnosis of pedal osteomyelitis in diabetic patients using current scintigraphic techniques. Arch Intern Med 1989; 149(10): 2262-6.

[21] Johnson JE, Kennedy EJ, Shereff MJ, Patel NC, Collier BD. Prospective study of bone, indium-111-labeled white blood cell, and gallium-67 scanning for the evaluation of osteomyelitis in the diabetic foot. Foot Ankle Int 1996; 17(1): 10-6.

[22] Newman LG, Waller J, Palestro CJ, et al. Leukocyte scanning with $111 \mathrm{In}$ is superior to magnetic resonance imaging in diagnosis of clinically unsuspected osteomyelitis in diabetic foot ulcers. Diabetes Care 1992; 15(11): 1527-30.

[23] Unal SN, Birinci H, Baktiroğlu S, Cantez S. Comparison of Tc99m methylene diphosphonate, Tc-99m human immune globulin, and Tc-99m-labeled white blood cell scintigraphy in the diabetic foot. Clin Nucl Med 2001; 26(12): 1016-21.

[24] Erdman WA, Buethe J, Bhore R, et al. Indexing severity of diabetic foot infection with 99mTc-WBC SPECT/CT hybrid imaging. Diabetes Care 2012; 35(9): 1826-31.

[25] Przybylski MM, Holloway S, Vyce SD, Obando A. Diagnosing osteomyelitis in the diabetic foot: A pilot study to examine the sensitivity and specificity of $\operatorname{Tc}(99 \mathrm{~m})$ white blood cell-labelled single photon emission computed tomography/computed tomography. Int Wound J 2016; 13(3): 382-9.

[26] Blume PA, Dey HM, Daley LJ, Arrighi JA, Soufer R, Gorecki GA. Diagnosis of pedal osteomyelitis with Tc-99m HMPAO labeled leukocytes. J Foot Ankle Surg 1997; 36(2): 120-6.

[27] Van der Wall H, Lunz D, Stanton F, Bruce W. Prognostic value of $99 \mathrm{~m}$ Tc leukocyte scintigraphy in diabetic pedal osteomyelitis. Foot Ankle Int 2001; 22(9): 720-4.

[28] Schauwecker DS, Park HM, Burt RW, Mock BH, Wellman HN. Combined bone scintigraphy and indium-111 leukocyte scans in neuropathic foot disease. J Nucl Med 1988; 29(10): 1651-5.

[29] Sanlı Y, Ozkan ZG, Unal SN, Türkmen C, Kılıçoğlu O. The Additional Value of Tc 99m HMPAO White Blood Cell SPECT in the Evaluation of Bone and Soft Tissue Infections. Mol Imaging Radionucl Ther 2011; 20(1): 7-13.

[30] Devillers A, Moisan A, Hennion F, Garin E, Poirier JY, Bourguet P. Contribution of technetium-99m hexamethylpropylene amine oxime labelled leucocyte scintigraphy to the diagnosis of diabetic foot infection. Eur J Nucl Med 1998; 25(2): 132-8.

[31] Heiba SI, Kolker D, Mocherla B, et al. The optimized evaluation of diabetic foot infection by dual isotope SPECT/CT imaging protocol. J Foot Ankle Surg 2010; 49(6): 529-36.

[32] Heiba S, Kolker D, Ong L, et al. Dual-isotope SPECT/CT impact on hospitalized patients with suspected diabetic foot infection: saving limbs, lives, and resources. Nucl Med Commun 2013; 34(9): 877-84.

[33] Ertugrul MB, Baktiroglu S, Salman S, et al. The diagnosis of osteomyelitis of the foot in diabetes: microbiological examination vs. magnetic resonance imaging and labelled leucocyte scanning. Diabet Med 2006; 23(6): 649-53.

[34] Larcos G, Brown ML, Sutton RT. Diagnosis of osteomyelitis of the foot in diabetic patients: value of $111 \mathrm{In}$-leukocyte scintigraphy. AJR Am J Roentgenol 1991; 157(3): 527-31.

[35] Vesco L, Boulahdour H, Hamissa S, et al. The value of combined radionuclide and magnetic resonance imaging in the diagnosis and conservative management of minimal or localized osteomyelitis of the foot in diabetic patients. Metabolism 1999; 48(7): 922-7.

[36] Newman LG, Waller J, Palestro CJ, et al. Unsuspected osteomyelitis in diabetic foot ulcers. Diagnosis and monitoring by leukocyte scanning with indium in 111 oxyquinoline. JAMA 1991; 266(9): 1246-51.

[37] Filippi L, Uccioli L, Giurato L, Schillaci O. Diabetic foot infection: usefulness of SPECT/CT for 99mTc-HMPAO-labeled leukocyte imaging. J Nucl Med 2009; 50(7): 1042-6.

[38] Familiari D, Glaudemans AW, Vitale V, et al. Can sequential 18FFDG PET/CT replace WBC imaging in the diabetic foot? J Nucl Med 2011; 52(7): 1012-9.

[39] Palestro CJ, Mehta HH, Patel M, et al. Marrow versus infection in the Charcot joint: indium-111 leukocyte and technetium-99m sulfur colloid scintigraphy. J Nucl Med 1998; 39(2): 346-50.

[40] Palestro CJ, Roumanas P, Swyer AJ, Kim CK, Goldsmith SJ. Diagnosis of musculoskeletal infection using combined In-111 labeled leukocyte and Tc-99m SC marrow imaging. Clin Nucl Med 1992; 17(4): 269-73.

[41] Tomas MB, Patel M, Marwin SE, Palestro CJ. The diabetic foot. Br J Radiol 2000; 73(868): 443-50.

[42] Vouillarmet J, Morelec I, Thivolet C. Assessing diabetic foot osteomyelitis remission with white blood cell SPECT/CT imaging. Diabet Med 2014; 31(9): 1093-9.

[43] Lazaga F, Van Asten SA, Nichols A, et al. Hybrid imaging with $99 \mathrm{mTc}-$ WBC SPECT/CT to monitor the effect of therapy in diabetic foot osteomyelitis. Int Wound J 2016; 13(6): 1158-60.

[44] Love C, Palestro CJ. Radionuclide imaging of infection. J Nucl Med Technol 2004; 32(2): 47-57.

[45] Dominguez-Gadea L, Martin-Curto LM, de la Calle H, Crespo A. Diabetic foot infections: scintigraphic evaluation with 99Tcmlabelled anti-granulocyte antibodies. Nucl Med Commun 1993; 14(3): 212-8.

[46] Palestro CJ, Caprioli R, Love C, et al. Rapid diagnosis of pedal osteomyelitis in diabetics with a technetium-99m-labeled monoclonal antigranulocyte antibody. J Foot Ankle Surg 2003; 42(1): 28 .

[47] Delcourt A, Huglo D, Prangere T, et al. Comparison between Leukoscan (Sulesomab) and Gallium-67 for the diagnosis of osteomyelitis in the diabetic foot. Diabetes Metab 2005; 31(2): 125-33.

[48] Harwood SJ, Valdivia S, Hung GL, Quenzer RW. Use of Sulesomab, a radiolabeled antibody fragment, to detect osteomyelitis in diabetic patients with foot ulcers by leukoscintigraphy. Clin Infect Dis 1999; 28(6): 1200-5.

[49] Heiba S, Knešaurek K. Evaluation of diabetic foot infection in nuclear medicine. Q J Nucl Med Mol Imaging 2017; 61(3): 283-91.

[50] Knešaurek K, Kolker D, Vatti S, Heiba S. Precise fusion of MRI and dual energy $111 \mathrm{In}$ WBC/99mTc HDP SPECT/CT in the diabetic foot using companion CT: An example of SPECT/MRI imaging. Q J Nucl Med Mol Imaging 2015; 59(1): 129-35.

[51] Auletta S, Baldoni D, Varani M, et al. Comparison of 99mTc-UBI 29-41, 99mTc-Ciprofloxacin, 99mTc-Ciprofloxacin dithiocarbamate and 111 In-biotin for targeting experimental Staphylococcus aureus and Escherichia coli foreign-body infections: An ex-vivo study. Q J Nucl Med Mol Imaging 2017; Epub ahead of print: http://dx.doi.org/10.23736/S1824-4785.17.02975-228849632 